\title{
História comparada do direito e seus desafios metodológicos*
}

\author{
Comparative legal history and its methodological challenges
}

Sylvain Soleil ${ }^{* *}$

\section{REFERÊNCIA}

SOLEIL, Sylvain. História comparada do direito e seus desafios metodológicos. Revista da Faculdade de Direito da UFRGS, Porto Alegre, n. 47, p. 03-20, dez. 2021. DOI: https://doi.org/10.22456/0104-6594.121033.

\begin{abstract}
RESUMO
Este artigo se apresenta como introdução a um conjunto de artigos fruto de conferências realizadas em Rennes, na França, onde se reuniram dez alunos de doutorado com Luisa Brunori, Jacques Bouineau e Sylvain Soleil. O autor indica dois desafios metodológicos em história comparada do direito: as carências metodológicas e a inibição comparativa. $\mathrm{O}$ caminho sugerido para superar os desafios é indicar, interrogar, bem como explicar e justificar a metodologia adotada; e, em outro plano, metamorfosear, transformar os problemas de comparabilidade e outros relacionados à comparação em benefícios para a pesquisa. Essa abordagem, denominada indutiva, enfatiza a prudência com que o autor deve escolher e fazer falar suas fontes, pensar a terminologia utilizada, conhecer e compreender o ambiente nos quais os direitos que se compara foram elaborados e possivelmente relacionados e, enfim, meditar sobre o significado e o interesse de sua comparação.
\end{abstract}

\section{PALAVRAS-CHAVE}

História do Direito. Direito comparado. Metodologia jurídica. Transplantes jurídicos.

\begin{abstract}
This article is presented as an introduction to a series of articles resulting from conferences held in Rennes, France, where ten doctoral students gathered with Luisa Brunori, Jacques Bouineau and Sylvain Soleil. The author points out two methodological challenges in comparative history of law: methodological shortcomings and comparative inhibition. The suggested way to overcome the challenges is to indicate, interrogate, as well as explain and justify the adopted methodology; and, on another level, to metamorphose and transform comparability problems and others related to comparison into benefits for research. This approach, called inductive, emphasizes the prudence with which the author must choose and have his sources speak, think about the adopted terminology, know and understand the environment in which the rights being compared were elaborated and possibly related, and, finally, meditate on the meaning and interest of its comparison.
\end{abstract}

\footnotetext{
* Texto original em língua francesa: SOLEIL, Sylvain. Les pièges, les méthodes, les ressources : Introduction. Historia et ius: rivista di storia giuridica dell'età medievale e mordena, [s.1.], n. 13, p. 02-12, 2018. ISSN 22797416. Appendice (Paper 17). Disponível em: <http://www.historiaetius.eu/num-13.html $>$. Tradução para a língua portuguesa por Alan Wruck Rangel (Pós-doutorando no PPGD-UERJ). Revisão da tradução por Alfredo de J. Flores (Professor Permanente do PPGD-UFRGS). Os tradutores agradecem a autorização dada pelo autor para esta publicação. O título em língua portuguesa aqui exposto foi sugerido pelo próprio autor, uma vez que o texto no original francês foi publicado dentro de um dossier, como texto introdutório. O autor da mesma forma apresentou o texto com as seguintes palavras: "Em dezembro de 2017, a conferência em Rennes (França) reuniu dez alunos de doutorado com Luisa Brunori, Jacques Bouineau e Sylvain Soleil. Eles apresentaram seus temas dentro dos termos da história comparada do direito e então discutiram sobre problemas de metodologia, desde um ponto de vista indutivo. Essa introdução apresenta a dupla mudança de método da história comparada do direito - a perspectiva indutiva que foi empregada durante a conferência e algumas fontes bibliográficas”. Os tradutores incluíram os títulos das seções 'Introdução' e 'Conclusão', divisão que não aparece no original. No texto original se encontra, nos elementos pós-textuais, uma seleção de referências a título de bibliografia essencial, juntada nesta versão no 'Anexo I - Lista de Bibliografia Essencial'.

** Professor de Introdução ao Direito e de História do Processo na Faculdade de Direito e de Ciência Política da Universidade de Rennes I, França. Pesquisador de Teoria e História dos Sistemas Jurídicos pela mesma instituição.
} 
KEYWORDS

Comparative Legal History. Comparative law. Legal methodology. Legal transplants.

\section{SUMÁRIO}

1. Introdução. 2. O duplo desafio do método em história comparada do direito. 3. O aporto de uma abordagem indutiva. 4. Conclusão. Referências. Anexo I - Lista de Bibliografia Essencial. Dados da publicação.

\section{INTRODUÇÃO}

Sem ser uma disciplina nova na França, a história comparada do direito ${ }^{a}$ foi por muito tempo prejudicada, isso apesar dos trabalhos fundadores, ou ainda alguns centros de pesquisa pioneiros (Lille, Nice, Aix-en-Provence etc.), ou também a evidência de um caráter complementar entre as abordagens histórica e comparativa ${ }^{1}$. Tal deficiência pode ser explicada de diversas maneiras. No plano histórico, ao usar a França menos do direito estrangeiro do que exportou, sem dúvida os historiadores do direito franceses sentiram menos a necessidade de fazer uso da comparação que seus colegas italianos, belgas, poloneses, romenos, japoneses ou latino-americanos. No plano acadêmico, os ensinamentos tradicionais das Faculdades de Direito priorizavam evidentemente a história do direito público e do direito privado francês, ao passo que os concursos públicos se concentravam mais na história do direito francês que na história jurídica europeia ou na história comparada do direito, o que, de modo mecânico, favorecia mais os trabalhos em direito francês que as teses e os artigos consagrados às fontes estrangeiras. Já desde o ponto de vista logístico, talvez fosse preciso também sublinhar as dificuldades que existiam, faz trinta ou mesmo vinte anos atrás, no que tange ao acesso à bibliografia, às fontes impressas e aos manuscritos estrangeiros, isso numa época em que a digitalização de textos e a formalização de redes europeias permaneceu pouco desenvolvida. Mas tudo isto ficou para trás. Juntam-se, aos trabalhos dos pioneiros, já faz vários anos, as pesquisas de novas gerações francesas de historiadores do direito ${ }^{2}$.

\footnotetext{
a Nota de tradução: o autor, Sylvain Soleil, faz referência à disciplina "Histoire Comparée du Droit", a qual, por sua vez, remete à nomenclatura já consolidada em língua inglesa na metodologia contemporânea que fala de uma "Comparative Legal History". Para a versão do termo em língua portuguesa, os tradutores optaram por utilizar a locução "História Comparada do Direito", mais fiel à terminologia francesa, mas que ainda não se consolidou na historiografia jurídica em língua portuguesa.

${ }^{1}$ Sobre o vínculo entre as duas disciplinas: HILAIRE, Jean. La place de l'histoire du droit dans l'enseignement et la formation du comparatiste. Revue Internationale de Droit Comparé, v. 50, n. 2, p. 319-333, 1998; TALON, D. Histoire du droit et droit comparé : une alliance féconde. In: BONTEMS, Claude. Nonagesimo Anno : mélanges en hommage à Jean Gaudemet. Paris: Presses Universitaires de France, 1999. p. 629 et seq.; EWALD, William. Legal History and Comparative Law. Zeitschrift für Europäisches Privatrecht, v. 7, n. 3, p. 553-559, 1999; GORDLEY, James. Comparative Law and Legal History. In: REIMANN, Mathias; ZIMMERMANN, Reinhard (ed.). The Oxford Handbook of Comparative Law. Oxford: Oxford University Press, 2006. p. 753 et seq.

${ }^{2}$ HALPÉRIN, Jean-Louis. Histoire du droit comparé. In: KRYNEN, Jacques; D’ALTEROCHE, Bernard (org.). L'histoire du droit en France: Nouvelles tendances, nouveaux territoires. Paris: Garnier, 2014. p. 183-203.
} 
No entanto, a questão do método ainda permanece porque, se os trabalhos se multiplicam (comparação de doutrinas, de legislações, de costumes...), isso ocorre sem o auxílio de uma metodologia bem identificada, bem pensada, bem avaliada. Por outro lado, vários autores e redes de pesquisa têm oferecido reflexões metodológicas úteis nos últimos anos (ver referências bibliográficas ao final do texto). E com razão: aos problemas específicos da história do direito se somam os da comparação do direito, o que constitui um duplo desafio.

\section{O DUPLO DESAFIO DO MÉTODO EM HISTÓRIA COMPARADA DO DIREITO}

A primeira armadilha para o historiador do direito seria não refletir suficientemente sobre o diálogo científico entre método histórico e método comparativo. As respectivas lógicas são complementares, mas seu ajuste não ocorre por si só. Um historiador do direito está sempre ciente de questões tais como: a comparabilidade das fontes; as palavras e os conceitos da comparação, da circulação, da imitação; a barreira da língua ou da cultura; a tradução (portanto, até certo ponto, a "traição") das fontes etc.? Está ele suficientemente armado desde o ponto de vista metodológico? Com isso, o método adotado é eficiente, confiável, ou mesmo legítimo, quando faz uma comparação de direitos? Heikki Pihlajamäki aborda essas questões por meio do posicionamento científico. Ele acredita que a história comparada do direito, à medida que se desenvolve, talvez se preocupe muito pouco em saber onde se situam as fronteiras entre as ciências (e, portanto, seus respectivos objetos e métodos):

[é] mais fácil [explica ele] adotar um ponto de vista liberal e se recusar a definir o método, a abordagem ou qualquer outro termo empregado para o designar. Se a abordagem 'deixa todas as flores desabrocharem' [let all the flowers bloom], ela é considerada como boa e simpática: por que se preocupar com limites e definições metodológicas, quando o que queremos são pesquisas pertinentes ${ }^{3}$.

Esta abordagem - insiste Heikki Pihlajamäki - por mais que seja boa e simpática, apesar de tudo, não pode se evadir de uma reflexão metodológica, porque, assim que o historiador do direito ultrapasse as fronteiras de seu campo inicial de competência, deve saber quem ele é e onde se encontra, para tirar o máximo de proveito das competências de seus vizinhos (sejam comparatistas, sociólogos, filósofos etc.), frustrando fenômenos de feudalização nas ciências.

\footnotetext{
${ }^{3}$ PIHLAJAMÄKI, Heikki. Comparative Context in Legal History: Are We All Comparatists Now?. In: ADAMS, Maurice; HEIRBAUT, Dirk (ed.). The method and culture of comparative law: essays in honour of Mark Van Hoecke. Oxford; Portland, Oregon: Hart Publishing, 2014. p. 121-132, p. 121. [Nota de tradução: o texto foi publicado em artigo de periódico brasileiro: PIHLAJAMÄKI, Heikki. Comparative Contexts in Legal History: are we all comparatists now?. Sequência, Florianópolis, n. 70, p. 57-75, 2015. DOI: <https://doi.org/10.5007/21777055.2015v36n70p57>].
} 
Em outras palavras: assim como um comparatista pode rapidamente - ao deixar de dominar as regras metodológicas essenciais da história do direito - aparecer como suspeito para os historiadores do direito, quando se aventura a fazer uma comparação de direitos recuando na história, por sua vez o historiador do direito também pode aparecer como suspeito, quando se aventura a fazer uma comparação de direitos, sem justificar seu método - por exemplo, a escolha de países, suas fontes, suas traduções. Martin Löhnig aborda, quanto a isso, a questão sob o ponto de vista dos objetivos (two ways - two goals?). Ele afirma, portanto, que:

a história do direito não tem verdadeiramente um método comparativo. Um historiador pode, desse modo, abrir a caixa de ferramentas do direito comparado e fazer uso '[d]a comparação histórica de legislações' ou empregar instrumentos de direito comparado e praticar uma 'história comparada do direito'4.

Daí emerge a questão sobre "o" método, porque - ainda segundo Martin Löhnig - existem duas formas de trabalhar, com objetivos que não são assimiláveis entre si, o que pode explicar por que razão a história comparada do direito ainda esteja em "fase de infância".

O primeiro desafio para o historiador do direito é, portanto, procurar conjugar o melhor possível o seu método histórico com o da comparação, o que pressupõe - voltaremos a isto adotar um método, saber questioná-lo e poder realizá-lo. É nesse sentido que Thomas Duve, ao reunir historiadores do direito preocupados com os fenômenos de "transferência ou transplante de normatividade" de um espaço territorial a outro, os convidava a se perguntar:

[q]uais são os métodos e as teorias que os historiadores utilizam para reconstruir os processos históricos de interação das diferentes ordens normativas? Por que utilizam esses conceitos e não outros? Quais são os pontos fortes e fracos dessas ferramentas metodológicas? ${ }^{5}$

Mas, para o historiador do direito, existe uma segunda armadilha que, paradoxalmente, seria muito preocupante a respeito da metodologia comparativa. Ele deve ser cauteloso quanto a isto, mas jamais além do razoável, para evitar de se perder ou ceder à inibição comparativa. Qualquer pesquisador interessado em história do direito comparado pode testemunhar que as controvérsias são consubstanciais ao direito comparado, e isto desde seu surgimento na virada dos séculos XIX e XX - assim: trata-se de uma ciência ou de um método? Qual critério deve

\footnotetext{
${ }^{4}$ LÖHNIG, Martin. Comparative Law and Legal History: a few words about Comparative Legal History. In: ADAMS, Maurice; HEIRBAUT, Dirk (ed.). The method and culture of comparative law: essays in honour of Mark Van Hoecke. Oxford; Portland, Oregon: Hart Publishing, 2014. p. 113-120, p. 113.

${ }^{5}$ DUVE, Thomas. Introduction: Entanglements in Legal History. Introductory Remarks. In: DUVE, Thomas (ed.). Entanglements in Legal History: Conceptual Approaches. Frankfurt: Max Planck Institute for European Legal History, 2014. p. 03-25, p. 05.
} 
ser adotado para classificar as várias formas de se fazer comparação jurídica? ${ }^{6} \mathrm{O}$ comparatista pode verdadeiramente isolar o regime jurídico de seu país, quando o compara com direitos estrangeiros? ${ }^{7}$ Podemos classificar os diversos sistemas jurídicos em famílias? ${ }^{8}$ Quais métodos de análise devem ser privilegiados para qualificar o processo de transplante, de empréstimo, de recepção, de transferência, de transposição, de aculturação? ${ }^{9}$ E etc. Portanto, fazer uma comparação de regimes jurídicos nos conduz necessariamente à exposição ante comentários,

${ }^{6}$ SOLEIL, Sylvain. Pourquoi comparait-on les droits au XIX'e siècle. Revue Clio@Themis, [s. l.], n. 13, p. 01-21, 2017. [Nota de tradução: tal texto de autoria de Soleil já foi traduzido: SOLEIL, Sylvain. Por que comparar os direitos no século XIX?. Tradução de Alan Wruck Rangel e Alfredo de J. Flores. Revista da Faculdade de Direito da UFRGS, Porto Alegre, n. 44, p. 182-228, dez. 2020].

${ }^{7}$ ZLATESCU, Victor Dan. Quelques aspects méthodologiques de la comparaison des droits. Revue Internationale de Droit Comparé, [s. l.], v. 35, n. 3, p. 559-566, 1983.

${ }^{8}$ ZAJTAY, Imre. Réflexions sur le problème de la division des familles de droits. Rabels Zeitschrift für ausländisches und internationales Privatrecht / The Rabel Journal of Comparative and International Private Law, [s. l.], v. 37, n. 2/3, p. 210-216, 1973; VANDERLINDEN, Jacques. À propos des familles de droit en droit civil comparé. In: Hommage à René Dekkers. Bruxelles: Bruylant, 1982. p. 352 et seq.; ROZMARYN, Stefan. Les grandes controverses du droit comparé. In: ROTONDI, Mario. Buts et méthodes du droit comparé. Padova: Cedam, 1973. p. 581 et seq. (Inchieste di diritto comparato, 4 vol., v. 2); MATTEI, Ugo. Verso una tripartizione non eurocentrica dei sistemi giuridici. In: Scintillae Iuris: studi in memoria di Gino Gorla. 3 vol. Milano: Giuffrè, 1994. v. 1, p. 776 et seq.; HUSA, Jaakko. Classification of Legal Families Today. Is it time for a memorial hymn?. Revue Internationale de Droit Comparé, v. 56, n. 1, p. 11-38, 2004.

${ }^{9}$ LEVY, Ernst. The Reception of Highly Developed Legal Systems by People of Different Cultures. Washington Law Review, v. 25, n. 3, p. 223 et seq., 1950; RHEINSTEIN, Max. Types of Reception. Annales de la Faculté de droit d'Istanbul, v. 5, n. 6, p. 31-40, 1956; ZAJTAY, Imre. La réception des droits étrangers et le droit comparé. Revue Internationale de Droit Comparé, v. 9, n. 4, p. 686-713, 1957; ALLIOT, Michel. L'acculturation juridique. In: POIRIER, Jean. (ed.). Ethnologie générale. Paris: Gallimard, 1968. p. 1180-1246; ANCEL, Marc. Utilité et méthodes du droit comparé: Éléments d'introduction générale à l'étude comparative des droits. Neuchâtel: Editions Ides et Calendes, 1971; RIVERO, Jean. Les phénomènes d'imitation des modèles étrangers en droit administratif. Bruxelles/Paris: Bruylant, 1972. p. 619 et seq. (Miscellanea Ganshof Van der Meersch. 3 vol., v. 3); CONSTANTINESCO, Léontin-Jean. Traité de droit comparé. 3 vol. Paris: Economica, 1972-1983; WATSON, Alan. Legal Transplants: An Approach to Comparative Law. Edinburgh: Scottish Academic Press, 1974; PAPACHRISTOS, Athanase. C. La réception des droits privés étrangers comme phénomène de sociologie juridique. Paris: LGDJ, 1975; GAUDEMET, Jean. Les transferts de droit. Année sociologique, v. 27, p. 29-59, 1976 (reprint : GAUDEMET, Jean. Les transferts de droit. In: GAUDEMET, Jean. Sociologie historique du droit. Paris: Presses Universitaires de France, 2000. p. 91 et seq.); RODIÈRE, René. Approche d'un phénomène: les migrations de systèmes juridiques. In: Mélanges dédiés à Gabriel Marty. Toulouse : Université des Sciences sociales de Toulouse, 1978. p. 947-954; AGOSTINI, Éric. La circulation des modèles juridiques. Revue Internationale de Droit Comparé, [s. l.], v. 42, n. 2, p. 461-467, 1990; MENY, Yves (ed.). Les politiques du mimétisme institutionnel : la greffe et le rejet. Paris: L'Harmattan, 1993; LEGRAND, Pierre. The impossibility of 'legal transplants'. Maastricht journal of European and comparative law, v. 4, n. 2, p. 111-124, 1997 [Nota de tradução: esse texto foi traduzido ao português - LEGRAND, Pierre. A impossibilidade de 'transplantes jurídicos'. Tradução de Gustavo Castagna Machado. Cadernos do PPGDir./UFRGS, vol. 09, n. 1, p. 01-21, 2014]; ROULAND, Norbert. Introduction historique au droit. Paris: Presses Universitaires de France, 1998; VELDE, Henk te. Political Transfer: An Introduction. European Review of History/ Revue européenne d'histoire, [s. l.], v. 12, n. 2, p. 205-221, 2005; SWIECICKA, Paulina. 'Connectiones inter iura variorum observamus...': En recherchant l'explication de la notion d'acculturation juridique. In: COPPEIN, Bart; STEVENS, Fred; WAELKENS, Laurent Leo Jozef Maria (ed.). Modernisme, tradition et acculturation juridique. Actes des Journées internationales de la Société d'histoire du droit. Bruxelles: Wetenschappelijk Comité voor Rechtsgeschiedenis, 2011. p. 07 et seq. (Iuris Scripta Historica XXVII); SOLEIL, Sylvain. La réception du modèle juridique français au XIX ${ }^{\mathrm{e}}$ siècle. Affaire de puissance ou de qualité technique?. In: COPPEIN, Bart; STEVENS, Fred; WAELKENS, Laurent Leo Jozef Maria (ed.). Modernisme, tradition et acculturation juridique. Actes des Journées internationales de la Société d'histoire du droit. Bruxelles: Wetenschappelijk Comité voor Rechtsgeschiedenis, 2011. p. 259 et seq. (Iuris Scripta Historica XXVII). 
debates, críticas, e até mesmo a acusações mais ou menos violentas. Nesse ponto, é importante compreender o impacto das reflexões epistemológicas recentes e buscar contextualizá-las. Por um lado, Otto Pfersmann, em 2001: “[...] o direito comparado suscitou imensas esperanças e ambiciosos empreendimentos, mas ainda se baseia na mais fraca das epistemologias" ${ }^{10}$. De outro, Marie-Claire Ponthoreau, em 2005: "Para a maioria dos comparatistas, o direito comparado é um método e não uma disciplina, mas eles não nos dizem nada ou muito pouco sobre o método em si" ${ }^{11}$. Por fim, Pascal Richard, em 2007:

[u]ma teoria do direito comparado está, de fato, muitas vezes ausente dos trabalhos dos comparatistas, embora esta disciplina - intimamente ligada ao seu objeto de estudo - deveria logicamente incluir um discurso sobre o seu método. A presença de tal carência epistemológica é difícil de justificar, mesmo quando alguns percebam no direito comparado não mais que $u m$ método ${ }^{12}$.

Essas acusações são suficientes para assustar os historiadores do direito que se aventuram a fazer uma comparação de direitos: quais condições, na realidade raramente ou jamais reunidas, seriam necessárias para poder comparar no espaço e no tempo esta ou aquela doutrina, esta ou aquela instituição, este ou aquele mecanismo jurídico? Por qual alquimia, qual elemento secundário do método se torna este tão crucial que corre o risco de invalidar toda a demonstração? Em suma, é possível fazer uma história comparada do direito? No entanto, essas acusações devem ser colocadas em seu contexto: todas essas reprovações estão formuladas para acompanhar o nascimento de uma nova ciência - a teoria ou a epistemologia do direito comparado - onde o objeto não é tanto a comparação de direitos em si senão uma reflexão sobre a maneira como se faz (ou não) a comparação dos direitos. Daí, ao mesmo tempo, o florescimento de estudos coletivos sobre este tema e suas diversas problematizações: por exemplo, os cadernos “Comparative Legal Studies: Traditions and transitions", publicados em 2000 por Pierre Legrand e Roderick Munday, durante o centenário do Congresso de Paris, para discutir as heranças sobre as quais se fundamentam os comparatistas: herança universalista (J. Gordley), colonial (U. Braxi), nacionalista (H. P. Glenn) ou funcionalista (M. Graziadei) ${ }^{13}$. Outro exemplo seria a obra "Epistemology and Methodology of Comparative Law", publicada

\footnotetext{
${ }^{10}$ PFERSMANN, Otto. Le droit comparé comme interprétation et comme théorie du droit. Revue Internationale de Droit Comparé, v. 53, n. 2, p. 275-288, 2001, p. 275.

${ }^{11}$ PONTHOREAU, Marie-Claire. Le droit comparé en question(s). Entre pragmatisme et outil épistémologique. Revue Internationale de Droit Comparé, v. 57, n. 1, p. 07-27, 2005, p. 07.

${ }^{12}$ RICHARD, Pascal. Le jeu de la différence. Réflexions sur l'épistémologie du droit comparé. Québec: Presses de l'Université Laval, 2007. p. 13.

${ }^{13}$ LEGRAND, Pierre; MUNDAY, Roderick J. C. (org.). Comparative legal studies: traditions and transitions. Cambridge: Cambridge Univ. Press, 2003. p. 31 et seq.
} 
em 2004, por Mark van Hoecke, com a finalidade de questionar as ferramentas tradicionais do direito comparado:

[e]nquanto muitos trabalhos modernos em direito comparado se concentram em vários aspectos da doutrina jurídica, o objetivo deste livro é de natureza mais teórica: refletir sobre o direito comparado como uma disciplina acadêmica, em particular sobre a sua epistemologia e metodologia ${ }^{14}$.

Trata-se, para o historiador do direito, de encontrar o distanciamento adequado desse conjunto de observações e dessa nova disciplina: se servir dela como um guia para evitar de escrever bobagens, mas sem se perder nela; encontrar elementos úteis em suas pesquisas, sem, para tanto, ceder à inibição.

\section{O APORTE DE UMA ABORDAGEM INDUTIVA}

Entre Charybde (as carências metodológicas) e Scylla (a inibição comparativa), o historiador do direito deve traçar sua trajetória. Como fazer isto? Por um lado, ele deve se amparar da questão metodológica, nomeá-la, tratá-la. Isso equivale, em primeiro lugar, a seguir um método - não só quando se seleciona o objeto da pesquisa, os países, as fontes e as palavras da comparação, mas também quando se traduz, quando se aproxima e diferencia, quando se tiram conclusões e se constrói uma exposição oral ou escrita. Se o direito comparado é um método, a história comparada do direito não deve nunca ocultar o método durante o processo de pesquisa: nem na escolha das fontes, nem na sua confrontação, nem na exposição dos dados, nem no desenvolvimento do plano etc. Para fazer isso, o historiador do direito dispõe de fontes e recomendações sob diversas formas: tratados, artigos, debates ${ }^{15}$. Trata-se, em seguida, de

\footnotetext{
${ }^{14}$ VAN HOECKE, Mark (ed.). Epistemology and Methodology of Comparative Law. Oxford: Bloomsbury, 2004. [Nota de tradução: na versão original em inglês: "Whereas many modern works on comparative law focus on various aspects of legal doctrine the aim of this book is of a more theoretical kind - to reflect on comparative law as a scholarly discipline, in particular at its epistemology and methodology."]

${ }^{15}$ ADAMS, Maurice; HEIRBAUT, Dirk (ed.). The method and culture of comparative law: essays in honour of Mark Van Hoecke. Oxford; Portland, Oregon: Hart Publishing, 2014; DUVE, Thomas. European Legal History Concepts, Methods, Challenges. In: DUVE, Thomas (ed.). Entanglements in Legal History: Conceptual Approaches. Frankfurt: Max Planck Institute for European Legal History, 2014. p. 29-66. [Nota de tradução: uma versão preliminar deste texto foi traduzida para o português: DUVE, Thomas. História do Direito Europeu Perspectivas Globais. Traduzido por Walter Guandalini Junior. Revista da Faculdade de Direito - UFPR, Curitiba, v. 60, n. 3, p. 383-412, 2015]; EWALD, William. Legal History and Comparative Law. Zeitschrift für Europäisches Privatrecht, v. 7, n. 3, p. 553-559, 1999; GORDLEY, James. Comparative Law and Legal History. In: REIMANN, Mathias; ZIMMERMANN, Reinhard (ed.). The Oxford Handbook of Comparative Law. Oxford: Oxford University Press, 2006. p. 753 et seq.; GRAZIADEI, Michele. Comparative Law, Legal History, and the Holistic Approach to Legal Cultures. Zeitschrift für Europäisches Privatrecht, v. 7, n. 3, p. 531 et seq., 1999; HALPÉRIN, Jean-Louis. Histoire du droit comparé. In: KRYNEN, Jacques; D’ALTEROCHE, Bernard (org.).
} 
interrogar o método à medida que a pesquisa avança, em particular quando se trata de pesquisa de longo prazo, para medir suas vantagens e pontos cegos, para dar maior abertura, melhor discussão e correção se necessário. Finalmente, convém explicar e justificar o método. Alguns usam a introdução, outros um capítulo preliminar. Algumas pessoas resumem as coisas em poucas frases, outras sentem a necessidade de longas explicações. Alguns explicam seu próprio método, outros apresentam os métodos possíveis antes de justificar sua escolha entre as alternativas. O principal disto tudo é explicar, prestar contas, justificar.

Por outro lado, deve-se saber transformar um problema em um benefício. Comecemos pelo exemplo de duas fontes que não parecem ser comparáveis; a do país A se apresenta sob a forma de legislações, a do país B sob a forma de costumes. Se o historiador do direito passa por cima e compara, termo por termo, determinado elemento do direito dos países A e B (por exemplo, um regime matrimonial, uma regra processual, um princípio constitucional) sem levar em conta a natureza das fontes, ele corre o risco de chegar a conclusões erradas. As razões são múltiplas e constituem o cerne da epistemologia e da teoria do direito comparado. Por outro lado, uma reflexão séria sobre o método pode permitir que se transcenda ao problema. Examinar-se-á a história do direito do primeiro país para entender e explicar por que a regra que se examina se apresenta sob a forma de legislação, e depois a do segundo país para estudar as razões pelas quais a regra vem na forma de costume. De modo que a comparação sobre o fundo do direito (as semelhanças e as diferenças entre as regras dos países A e B) seja enriquecida por uma comparação sobre a evolução do direito nos dois países: por que o país A substituiu o costume pela legislação? Essa substituição teve consequências sobre a publicidade

L'histoire du droit en France : Nouvelles tendances, nouveaux territoires. Paris: Garnier, 2014. p. 183-203; LUIG, Klaus. Was kann die Rechtsgeschichte der Rechtsvergleichung bieten?. Zeitschrift für Europäisches Privatrecht, v. 7, n. 3, p. 521 et seq., 1999; MUSSON, Anthony; STEBBINGS, Chantal (ed.). Making Legal History: Approaches and Methodologies. Cambridge: Cambridge University Press, 2012; REINMANN, Mathias. Rechtsvergleichung und Rechtsgeschichte im Dialog. Zeitschrift für Europäisches Privatrecht, v. 7, n. 3, p. 496 et seq., 1999; ZIMMERMANN, Reinhard. L'héritage de Savigny. Histoire du droit, droit comparé, et émergence d'une science juridique européenne. Revue internationale de droit économique, [s. l.], t. XXVII, n. 1-2, p. 95-127, 2013. A essa bibliografia, convém acrescentar uma recente publicação: MORÉTEAU, Olivier; MASFERRER, Aniceto; MODÉER, Kjell. A. (ed.). Comparative Legal History. Cheltenham: Edward Elgar Publishing, 2018. onde os autores, falando tanto do fundo como do método, rogam por uma constituição da história comparada do direito como disciplina de forma integral, com, evidentemente, reflexões de natureza metodológica. A esses títulos, convém, ainda, acrescentar (essas últimas orientações bibliográficas foram indicadas por Michel Morin, a quem se deve agradecer): IBBETSON, David. The Challenges of Comparative Legal History. Comparative Legal History, [s. l.], v. 1, n. 1, p. 01-11, 2013; DUVE, Thomas. Legal traditions: A dialogue between comparative law and comparative legal history. Comparative Legal History, [s. l.], v. 6, n. 1, p. 15-33, 2018; DAUCHY, Serge. Ouverture: Histoires des cultures juridiques. Circulations, connexions et espaces transnationaux du droit. Revue Clio@Themis, [s. l.], n. 2, 2021; PARISE, Agustín. Ownership paradigms in American civil law jurisdictions: manifestations of the shifts in the legislation of Louisiana, Chile, and Argentina (16th-20th centuries). Leiden: Brill, 2017; CAIRNS, John W. Codification, transplants and history: law reform in Louisiana (1808) and Quebec (1866). Clark, New Jersey: Talbot Publishing, 2015. 
da norma, sobre o seu conteúdo, sobre a uniformização do direito etc.? Por que o país B conservou os costumes? É por respeito à tradição jurídica? É por rejeição à lei? É pela recusa à imitação de um direito estrangeiro? A partir de então, a comparação histórica dos direitos assume sua verdadeira dimensão. Não é mais uma questão de comparar as regras jurídicas de dois países em um momento dado. Já não mais se trata de examinar dois conjuntos que, do jeito que estão, não são realmente comparáveis. Trata-se de comparar dois elementos jurídicos em sua evolução histórica: um que se apresenta na forma de legislação; o outro que se apresenta na forma de costumes - assim, um e outro ficam esclarecidos por um conjunto de fatores antropológicos, políticos, econômicos etc.; um e outro são problematizados por um conjunto de questões. O problema da comparabilidade das fontes não foi contornado. Ele foi metamorfoseado.

Estas duas lógicas (Apreender a questão metodológica / Saber transformar um problema em um beneficio) estão na base da abordagem indutiva preconizada durante a jornada de estudos realizada em Rennes, em dezembro de 2017. Dez doutorandos em história do direito se reuniram, sob a coordenação de Sylvain Soleil (Universidade de Rennes 1), Luisa Brunori (CNRS - Universidade de Lille 2) e Jacques Bouineau (Universidade La Rochelle), graças à rede e à força de persuasão de Hugo Beuvant, aluno de doutorado na Universidade Rennes 1. Todos foram convidados a apresentar seu objeto de pesquisa, a identificar um problema específico vinculado à comparação de direitos, a explicar a solução preconizada e a submetê-la à discussão.

Claire de Blois ${ }^{\mathrm{b}}$, doutoranda da Universidade de Paris V, enfoca as dificuldades postas pelo conceito de "fronteira" nos tratados internacionais, a partir do exemplo do tratado dos Pirineus. Para comparar a maneira como os autores, os diplomatas e os agentes franceses e estrangeiros concebiam a fronteira (seu significado, sua definição, seu conteúdo, sua delimitação), o problema maior dizia respeito à semântica: se os substantivos (fronteira, limite, demarcação, confins), os verbos ou os adjetivos não têm o mesmo significado de um lado e do outro da fronteira, a comparação não alcançaria o próprio objeto da comparação? Como comparar se os países vizinhos, por trás das palavras, não compartilham a concepção francesa de fronteira? A autora questionou, portanto, o seu método ao optar por abordar seu objeto de

\footnotetext{
${ }^{\mathrm{b}}$ Nota de tradução: o texto da autora teve sua publicação junto ao mesmo dossier que o original deste artigo se faz presente, assim como os mencionados nas notas seguintes - BLOIS, Claire de. Les difficultés posées par la délimitation de la frontière au XVII ${ }^{\mathrm{e}}$ siècle : la confrontation du concept français et espagnol. Historia et ius: rivista di storia giuridica dell'età medievale e mordena, [s.1.], n. 13, p. 13-21, 2018. ISSN 2279-7416. Appendice (Paper 17). Disponível em: <http://www.historiaetius.eu/num-13.html>.
} 
pesquisa partindo do estudo do sentido das palavras, visto que podem ser enganosas; depois, pelos procedimentos concretos previstos nos tratados de paz, a partir dos quais os comissários franceses e estrangeiros delimitam dois territórios (listas de entidades territoriais em disputa, critérios para fixar uma linha divisória, demarcação). Em seguida, retorna ao vocabulário para identificar, nos tratados de paz, os substantivos em língua estrangeira que correspondem aos substantivos franceses "fronteira" e "limite". A dificuldade metodológica não foi evitada; ela permitiu aprofundar a substância da pesquisa comparativa.

Romain Broussais ${ }^{\mathrm{c}}$, doutorando na Universidade de Paris II, dedica seu estudo à comparação dos estatutos urbanos medievais no reino da França. A dificuldade do seu objeto de pesquisa reside na diversidade de abordagens jurídicas e institucionais medievais: a "França urbana", explica ele:

é composta, do lado oeste, pelas cidades dos Établissements de Rouen; no norte, pelo movimento communal picard e pelo movimento échevinal flamand; no lado leste, pelo échevinat, igualmente, mas também pelo estatuto imperial de certas cidades; ao sul, pelas villes de consulats, [...].

Isso coloca um grande problema de comparabilidade das fontes: as regras resultantes dos estatutos são comparáveis e, em caso afirmativo, como? Podemos aproximar o regulamento urbano (elaborado pelos burgueses) a uma carta concedida por um senhor? O autor, portanto, decidiu não seguir uma abordagem que iria de cima (os estatutos) para baixo (o conteúdo das próprias regras), mas seguir uma abordagem invertida: "comparar a partir do particular - as disposições normativas - para ir em direção ao mais geral - os conjuntos jurídicos”. Ele explica esse processo por meio de um círculo de comparação, complexo e abrangente, que lhe permite criar conjuntos jurídicos comparáveis. O problema metodológico permitiu ao autor considerar suas fontes e seu objeto de pesquisa sob uma nova perspectiva.

Elodie Coutant ${ }^{\mathrm{d}}$, doutoranda na Universidade de Bordéus, examina a reforma do direito francês sob o prisma do direito local da Alsácia-Mosela, ou seja:

um conjunto de disposições alemãs; de disposições francesas anteriores a 1871 mantidas pelas autoridades alemãs; de regras adotadas pelas instituições alsacianas e lorenas; e das disposições francesas posteriores a 1918 e aplicáveis nesses territórios.

\footnotetext{
c Nota de tradução: BROUSSAIS, Romain. Comparer les statuts urbains médiévaux. Historia et ius: rivista di storia giuridica dell'età medievale e mordena, [s.1.], n. 13, p. 22-28, 2018. ISSN 2279-7416. Appendice (Paper 17). Disponível em: $<$ http://www.historiaetius.eu/num-13.html $>$.

d Nota de tradução: COUTANT, Elodie. L'étude de la réforme du droit français à travers le prisme du droit local alsacien-mosellan: la nécessité d'une méthode sur-mesure. Historia et ius: rivista di storia giuridica dell'età medievale e mordena, [s.1.], n. 13, p. 29-37, 2018. ISSN 2279-7416. Appendice (Paper 17). Disponível em: $<$ http://www.historiaetius.eu/num-13.html $>$.
} 
Numa primeira abordagem, o seu objeto de investigação esbarra tanto na diluição progressiva desse direito no direito francês e na vontade política ou social de minimizar ou, pelo contrário, exagerar as diferenças entre o direito alsaciano-moselano e o direito da República, a qual é una e indivisível. A autora, em vez de criar artificialmente dois conjuntos jurídicos para comparálos, decidiu, portanto, adotar uma segunda abordagem, histórica e sociológica, para colocar em questão os verdadeiros desafios: quem, quando, como e por que se tem interesse em insistir na originalidade do direito local? Quem, ao contrário, tem interesse em fundir o direito local no direito geral? Etc. Explica a autora: “[é] importante não compor com essas dificuldades, mas, antes, explorá-las. São de fato significativas para o período e para a percepção que podemos ter sobre o direito local e a reforma do direito francês. São, até certo ponto, o cerne de nossa análise". Em outras palavras, a dificuldade metodológica está no cerne da própria tese. Bem identificado, torna-se a questão central.

Théa Dougbo ${ }^{\mathrm{e}}$ dedica seu estudo à comparação entre regimes dotais de direito comum na Europa e na África Ocidental nas vésperas da colonização. A priori, a comparação aparece não só legítima, mas também bastante fácil de imaginar: se, em ambos os casos, trata-se de situar a união dos cônjuges na união de duas linhagens, entrelaçando vínculo matrimonial e vínculo patrimonial, duas lógicas antropológicas e históricas parecem estar em ação, dependendo se o dote é pago pelo marido (ou sua família) à família da esposa (lógica africana), ou pela família da esposa em benefício do lar (lógica europeia). A dificuldade surge da distorção entre as fontes: algumas (europeias) são na sua maioria codificadas no século XIX, enquanto outras (africanas) são principalmente conhecidas graças à antropologia contemporânea. Os dois conjuntos são difíceis de comparar. O autor optou, portanto, por pesquisar os costumes, em matéria de dotes, nos compêndios que foram elaborados pelos administradores coloniais no início do século XX, o que muda o cerne da comparação. Se esses compêndios oferecem dados escritos e exploráveis, eles arrolam os costumes comparando-os com o que os administradores coloniais conheciam (as legislações europeias) e, ao fazê-lo, modificam parcialmente os costumes estudados: eles foram colocados por escrito com palavras, definições, categorias, regimes jurídicos franceses e, de forma mais geral, pelas formas de pensar sobre o casamento na Europa. A dificuldade metodológica leva, assim, o autor a modificar e enriquecer seu objeto

\footnotetext{
${ }^{\text {e }}$ Nota de tradução: DOUGBO, Théa Martial. Les régimes dotaux de droit commun en Europe et en Afrique de l'ouest à la veille de la colonisation. Le problème de la comparabilité des sources. Historia et ius: rivista di storia giuridica dell'età medievale e mordena, [s.1.], n. 13, p. 38-41, 2018. ISSN 2279-7416. Appendice (Paper 17). Disponível em: $<$ http://www.historiaetius.eu/num-13.html $>$.
} 
inicial de pesquisa. A comparação histórica desliza em direção à comparação entre o regime dotal de direito comum da Europa no século XIX e o regime de direito comum da África conforme percebido, traduzido e transcrito pelos administradores coloniais.

\section{CONCLUSÃO}

Essa abordagem indutiva, que parte dos problemas de método tal como foram identificados pelos autores e as soluções por eles concebidas e que oferecem à discussão, conduz a reflexões, conselhos, regras gerais e advertências, que Luisa Brunori ${ }^{\mathrm{f}}$ sintetizou em um "decálogo" que serve, aqui, tanto como conclusão como como guia. Enfatiza em particular a prudência com que o autor deve escolher e fazer falar suas fontes, deve pensar sobre a terminologia utilizada, deve conhecer e compreender o ambiente nos quais os direitos que se compara foram elaborados, e possivelmente relacionados, deve, enfim, meditar sobre o significado e interesse de sua comparação.

\section{REFERÊNCIAS}

ADAMS, Maurice; HEIRBAUT, Dirk (ed.). The method and culture of comparative law: essays in honour of Mark Van Hoecke. Oxford; Portland, Oregon: Hart Publishing, 2014.

AGOSTINI, Éric. La circulation des modèles juridiques. Revue Internationale de Droit Comparé, [s.l.], v. 42, n. 2, p. 461-467, 1990.

ALLIOT, Michel. L'acculturation juridique. In: POIRIER, Jean. (ed.). Ethnologie générale. Paris: Gallimard, 1968. p. 1180-1246.

ANCEL, Marc. Utilité et méthodes du droit comparé: Éléments d'introduction générale à l'étude comparative des droits. Neuchâtel: Editions Ides et Calendes, 1971.

CAIRNS, John W. Codification, transplants, and history: law reform in Louisiana (1808) and Quebec (1866). Clark, New Jersey: Talbot Publishing, 2015.

CONSTANTINESCO, Léontin-Jean. Traité de droit comparé. 3 vol. Paris: Economica, 19721983.

DAUCHY, Serge. Ouverture: Histoires des cultures juridiques. Circulations, connexions et espaces transnationaux du droit.RevueClio@Themis, [s. l.], n. 2, 2021.

${ }^{\dagger}$ Nota de tradução: BRUNORI, Luisa. Le « décalogue » de l'historien du droit comparatiste. Historia et ius: rivista di storia giuridica dell'età medievale e mordena, [s.1.], n. 13, p. 42-47, 2018. ISSN 2279-7416. Disponível em: $<$ http://www.historiaetius.eu/num-13.html $>$. 
DUVE, Thomas. European Legal History - Concepts, Methods, Challenges. In: DUVE, Thomas (ed.). Entanglements in Legal History: Conceptual Approaches. Frankfurt: Max Planck Institute for European Legal History, 2014. p. 29-66.

DUVE, Thomas. Introduction: Entanglements in Legal History. Introductory Remarks. In: DUVE, Thomas (ed.). Entanglements in Legal History: Conceptual Approaches. Frankfurt: Max Planck Institute for European Legal History, 2014. p. 03-25.

DUVE, Thomas. Legal traditions: A dialogue between comparative law and comparative legal history. Comparative Legal History, [s. l.], v. 6, n. 1, p. 15-33, 2018.

EWALD, William. Legal History and Comparative Law. Zeitschrift für Europäisches Privatrecht, v. 7, n. 3, p. 553-559, 1999.

GAUDEMET, Jean. Les transferts de droit. Année sociologique, v. 27, p. 29-59, 1976.

GAUDEMET, Jean. Les transferts de droit. In: GAUDEMET, Jean. Sociologie historique du droit. Paris: Presses Universitaires de France, 2000. p. 91 et seq.

GORDLEY, James. Comparative Law and Legal History. In: REIMANN, Mathias; ZIMMERMANN, Reinhard (ed.). The Oxford Handbook of Comparative Law. Oxford: Oxford University Press, 2006. p. 753 et seq.

GRAZIADEI, Michele. Comparative Law, Legal History, and the Holistic Approach to Legal Cultures. Zeitschrift für Europäisches Privatrecht, v. 7, n. 3, p. 531 et seq., 1999.

HALPÉRIN, Jean-Louis. Histoire du droit comparé. In: KRYNEN, Jacques; D’ALTEROCHE, Bernard (org.). L'histoire du droit en France : Nouvelles tendances, nouveaux territoires. Paris: Garnier, 2014. p. 183-203.

HILAIRE, Jean. La place de l'histoire du droit dans l'enseignement et la formation du comparatiste. Revue Internationale de Droit Comparé, v. 50, n. 2, p. 319-333, 1998.

HUSA, Jaakko. Classification of Legal Families Today. Is it time for a memorial hymn?. Revue Internationale de Droit Comparé, v. 56, n. 1, p. 11-38, 2004.

IBBETSON, David. The Challenges of Comparative Legal History. Comparative Legal History, [s. l.], v. 1, n. 1, p. 01-11, 2013.

LEGRAND, Pierre. The impossibility of 'legal transplants'. Maastricht journal of European and comparative law, v. 4, n. 2, p. 111-124, 1997.

LEGRAND, Pierre; MUNDAY, Roderick J. C. (org.). Comparative legal studies: traditions and transitions. Cambridge: Cambridge Univ. Press, 2003.

LEVY, Ernst. The Reception of Highly Developed Legal Systems by People of Different Cultures. Washington Law Review, v. 25, n. 3, p. 223 et seq., 1950. 
LÖHNIG, Martin. Comparative Law and Legal History: a few words about Comparative Legal History. In: ADAMS, Maurice; HEIRBAUT, Dirk (ed.). The method and culture of comparative law: essays in honour of Mark Van Hoecke. Oxford; Portland, Oregon: Hart Publishing, 2014. p. 113-120.

LUIG, Klaus. Was kann die Rechtsgeschichte der Rechtsvergleichung bieten?. Zeitschrift für Europäisches Privatrecht, v. 7, n. 3, p. 521 et seq., 1999.

MATTEI, Ugo. Verso una tripartizione non eurocentrica dei sistemi giuridici. In: Scintillae Iuris: studi in memoria di Gino Gorla. 3 vol. Milano: Giuffrè, 1994. v. 1, p. 776 et seq.

MENY, Yves (ed.). Les politiques du mimétisme institutionnel: la greffe et le rejet. Paris: L'Harmattan, 1993.

MORÉTEAU, Olivier; MASFERRER, Aniceto; MODÉER, Kjell. A. (ed.). Comparative Legal History. Cheltenham: Edward Elgar Publishing, 2018.

MUSSON, Anthony; STEBBINGS, Chantal (ed.). Making Legal History: Approaches and Methodologies. Cambridge: Cambridge University Press, 2012.

PAPACHRISTOS, Athanase. C. La réception des droits privés étrangers comme phénomène de sociologie juridique. Paris: LGDJ, 1975.

PARISE, Agustín. Ownership paradigms in American civil law jurisdictions: manifestations of the shifts in the legislation of Louisiana, Chile, and Argentina (16th-20th centuries). Leiden: Brill, 2017.

PFERSMANN, Otto. Le droit comparé comme interprétation et comme théorie du droit. Revue Internationale de Droit Comparé, v. 53, n. 2, p. 275-288, 2001.

PIHLAJAMÄKI, Heikki. Comparative Context in Legal History: Are We All Comparatists Now?. In: ADAMS, Maurice; HEIRBAUT, Dirk (ed.). The method and culture of comparative law: essays in honour of Mark Van Hoecke. Oxford; Portland, Oregon: Hart Publishing, 2014. p. 121-132.

PONTHOREAU, Marie-Claire. Le droit comparé en question(s). Entre pragmatisme et outil épistémologique. Revue Internationale de Droit Comparé, v. 57, n. 1, p. 07-27, 2005.

REINMANN, Mathias. Rechtsvergleichung und Rechtsgeschichte im Dialog. Zeitschrift für Europäisches Privatrecht, v. 7, n. 3, p. 496 et seq., 1999.

RHEINSTEIN, Max. Types of Reception. Annales de la Faculté de droit d'Istanbul, v. 5, n. 6, p. $31-40,1956$.

RICHARD, Pascal. Le jeu de la différence. Réflexions sur l'épistémologie du droit comparé. Québec: Presses de 1’Université Laval, 2007. 
RIVERO, Jean. Les phénomènes d'imitation des modèles étrangers en droit administratif. Bruxelles/Paris: Bruylant, 1972. p. 619 et seq. (Miscellanea Ganshof Van der Meersch. 3 vol., v. 3).

RODIÈRE, René. Approche d'un phénomène: les migrations de systèmes juridiques. In: Mélanges dédiés à Gabriel Marty. Toulouse: Université des Sciences sociales de Toulouse, 1978. p. 947-954.

ROULAND, Norbert. Introduction historique au droit. Paris: Presses Universitaires de France, 1998.

ROZMARYN, Stefan. Les grandes controverses du droit comparé. In: ROTONDI, Mario. Buts et méthodes du droit comparé. Padova: Cedam, 1973. p. 581 et seq. (Inchieste di diritto comparato, 4 vol., v. 2).

SOLEIL, Sylvain. La réception du modèle juridique français au XIX ${ }^{\mathrm{e}}$ siècle. Affaire de puissance ou de qualité technique?. In: COPPEIN, Bart; STEVENS, Fred; WAELKENS, Laurent Leo Jozef Maria (ed.). Modernisme, tradition et acculturation juridique. Actes des Journées internationales de la Société d'histoire du droit. Bruxelles: Wetenschappelijk Comité voor Rechtsgeschiedenis, 2011. p. 259 et seq. (Iuris Scripta Historica XXVII).

SOLEIL, Sylvain. Pourquoi comparait-on les droits au XIX ${ }^{\mathrm{e}}$ siècle.Revue Clio@Themis, [s. l.], n. 13, p. 01-21, 2017.

SWIECICKA, Paulina. 'Connectiones inter iura variorum observamus...': En recherchant l'explication de la notion d'acculturation juridique. In: COPPEIN, Bart; STEVENS, Fred; WAELKENS, Laurent Leo Jozef Maria (ed.). Modernisme, tradition et acculturation juridique. Actes des Journées internationales de la Société d'histoire du droit. Bruxelles: Wetenschappelijk Comité voor Rechtsgeschiedenis, 2011. p. 07 et seq. (Iuris Scripta Historica XXVII).

TALON, D. Histoire du droit et droit comparé: une alliance féconde. In: BONTEMS, Claude. Nonagesimo Anno: mélanges en hommage à Jean Gaudemet. Paris: Presses Universitaires de France, 1999. p. 629 et seq.

VAN HOECKE, Mark (ed.). Epistemology and Methodology of Comparative Law. Oxford: Bloomsbury, 2004.

VANDERLINDEN, Jacques. À propos des familles de droit en droit civil comparé. In: Hommage à René Dekkers. Bruxelles: Bruylant, 1982. p. 352 et seq.

VELDE, Henk te. Political Transfer: An Introduction. European Review of History / Revue européenne d'histoire, [s. l.], v. 12, n. 2, p. 205-221, 2005.

WATSON, Alan. Legal Transplants: An Approach to Comparative Law. Edinburgh: Scottish Academic Press, 1974.

ZAJTAY, Imre. La réception des droits étrangers et le droit comparé. Revue Internationale de Droit Comparé, v. 9, n. 4, p. 686-713, 1957. 
ZAJTAY, Imre. Réflexions sur le problème de la division des familles de droits. Rabels Zeitschrift für ausländisches und internationales Privatrecht / The Rabel Journal of Comparative and International Private Law, [s. l.], v. 37, n. 2/3, p. 210-216, 1973.

ZIMMERMANN, Reinhard. L'héritage de Savigny. Histoire du droit, droit comparé, et émergence d'une science juridique européenne. Revue internationale de droit économique, [s. l.], t. XXVII, n. 1-2, p. 95-127, 2013.

ZLATESCU, Victor Dan. Quelques aspects méthodologiques de la comparaison des droits. Revue internationale de droit comparé, [s. l.], v. 35, n. 3, p. 559-566, 1983.

\section{REFERÊNCIAS EM NOTAS DE TRADUÇÃO}

BLOIS, Claire de. Les difficultés posées par la délimitation de la frontière au XVII ${ }^{\mathrm{e}}$ siècle: la confrontation du concept français et espagnol. Historia et ius: rivista di storia giuridica dell'età medievale e moderna, [s.1.], n. 13, p. 13-21, 2018. ISSN 2279-7416. Appendice (Paper 17). Disponível em: <http://www.historiaetius.eu/num-13.html $>$.

BROUSSAIS, Romain. Comparer les statuts urbains médiévaux. Historia et ius: rivista di storia giuridica dell'età medievale e moderna, [s.1.], n. 13, p. 22-28, 2018. Appendice (Paper 17). ISSN 2279-7416. Disponível em: <http://www.historiaetius.eu/num-13.html>.

BRUNORI, Luisa. Le « décalogue » de l'historien du droit comparatiste. Historia et ius: rivista di storia giuridica dell'età medievale e moderna, [s.1.], n. 13, p. 42-47, 2018. ISSN 2279-7416. Appendice (Paper 17). Disponível em: <http://www.historiaetius.eu/num-13.html $>$.

COUTANT, Elodie. L'étude de la réforme du droit français à travers le prisme du droit local alsacien-mosellan : la nécessité d'une méthode sur-mesure. Historia et ius: rivista di storia giuridica dell'età medievale e moderna, [s.1.], n. 13, p. 29-37, 2018. Appendice (Paper 17). ISSN 2279-7416. Disponível em: <http://www.historiaetius.eu/num-13.html>.

DOUGBO, Théa Martial. Les régimes dotaux de droit commun en Europe et en Afrique de l'ouest à la veille de la colonisation. Le problème de la comparabilité des sources. Historia et ius: rivista di storia giuridica dell'età medievale e moderna, [s.1.], n. 13, p. 38-41, 2018. ISSN 2279-7416. Appendice (Paper 17). Disponível em: <http://www.historiaetius.eu/num13.html>.

DUVE, Thomas. História do Direito Europeu - Perspectivas Globais. Tradução de Walter Guandalini Junior. Revista da Faculdade de Direito - UFPR, Curitiba, v. 60, n. 3, p. 383-412, 2015.

LEGRAND, Pierre. A impossibilidade de 'transplantes jurídicos'. Tradução de Gustavo Castagna Machado. Cadernos do PPGDir./UFRGS, Porto Alegre, v. 09, n. 1, p. 01-21, 2014.

SOLEIL, Sylvain. Por que comparar os direitos no século XIX?. Tradução de Alan Wruck Rangel e Alfredo de J. Flores. Revista da Faculdade de Direito da UFRGS, Porto Alegre, n. 44, p. 182-228, dez. 2020. 
SOLEIL, Sylvain. Les pièges, les méthodes, les ressources : Introduction. Historia et ius: rivista di storia giuridica dell'età medievale e moderna, [s.1.], n. 13, p. 02-12, 2018. ISSN 2279-7416. Appendice (Paper 17). Disponível em: <http://www.historiaetius.eu/num-13.html $>$.

PIHLAJAMÄKI, Heikki. Comparative Contexts in Legal History: are we all comparatists now?. Sequência, Florianópolis, n. 70, p. 57-75, 2015. DOI: <https://doi.org/10.5007/21777055.2015v36n70p57>.

\section{ANEXO I - LISTA DE BIBLIOGRAFIA ESSENCIAL}

ADAMS, Maurice; HEIRBAUT, Dirk (ed.). The method and culture of comparative law: essays in honour of Mark Van Hoecke. Oxford; Portland, Oregon: Hart Publishing, 2014.

CAIRNS, John W. Codification, transplants, and history: law reform in Louisiana (1808) and Quebec (1866). Clark, New Jersey: Talbot Publishing, 2015.

CONSTANTINESCO, Léontin-Jean. Traité de droit comparé. 3 vol. Paris: Economica, 19721983.

DAUCHY, Serge. Ouverture: Histoires des cultures juridiques. Circulations, connexions et espaces transnationaux du droit.Revue Clio@Themis, [s. l.], n. 2, 2021.

DUVE, Thomas. European Legal History - Concepts, Methods, Challenges. In: DUVE, Thomas (ed.). Entanglements in Legal History: Conceptual Approaches. Frankfurt: Max Planck Institute for European Legal History, 2014. p. 29-66.

DUVE, Thomas. Legal traditions: A dialogue between comparative law and comparative legal history. Comparative Legal History, [s. l.], v. 6, n. 1, p. 15-33, 2018.

EWALD, William. Legal History and Comparative Law. Zeitschrift für Europäisches Privatrecht, v. 7, n. 3, p. 553-559, 1999.

GORDLEY, James. Comparative Law and Legal History. In: REIMANN, Mathias; ZIMMERMANN, Reinhard (ed.). The Oxford Handbook of Comparative Law. Oxford: Oxford University Press, 2006. p. 753 et seq.

GRAZIADEI, Michele. Comparative Law, Legal History, and the Holistic Approach to Legal Cultures. Zeitschrift für Europäisches Privatrecht, v. 7, n. 3, p. 531 et seq., 1999.

HALPÉRIN, Jean-Louis. Histoire du droit comparé. In: KRYNEN, Jacques; D’ALTEROCHE, Bernard (org.). L'histoire du droit en France: Nouvelles tendances, nouveaux territoires. Paris: Garnier, 2014. p. 183-203.

HILAIRE, Jean. La place de l'histoire du droit dans l'enseignement et la formation du comparatiste. Revue Internationale de Droit Comparé, v. 50, n. 2, p. 319-333, 1998. 
IBBETSON, David. The Challenges of Comparative Legal History. Comparative Legal History, [s. l.], v. 1, n. 1, p. 01-11, 2013.

LEGRAND, Pierre. Le droit comparé. Paris: Presses Universitaires de France, 1999.

LEGRAND, Pierre; MUNDAY, Roderick J. C. (org.). Comparative legal studies: traditions and transitions. Cambridge: Cambridge Univ. Press, 2003.

LUIG, Klaus. Was kann die Rechtsgeschichte der Rechtsvergleichung bieten?. Zeitschrift für Europäisches Privatrecht, v. 7, n. 3, p. 521 et seq., 1999.

MORÉTEAU, Olivier; MASFERRER, Aniceto; MODÉER, Kjell. A. (ed.). Comparative Legal History. Cheltenham: Edward Elgar Publishing, 2018.

MUSSON, Anthony; STEBBINGS, Chantal (ed.). Making Legal History: Approaches and Methodologies. Cambridge: Cambridge University Press, 2012.

PARISE, Agustin. Ownership paradigms in American civil law jurisdictions: manifestations of the shifts in the legislation of Louisiana, Chile, and Argentina (16th-20th centuries). Leiden: Brill, 2017.

REINMANN, Mathias. Rechtsvergleichung und Rechtsgeschichte im Dialog. Zeitschrift für Europäisches Privatrecht, v. 7, n. 3, p. 496 et seq., 1999.

SACCO, Rodolfo. La comparaison juridique au service de la connaissance du droit. Paris: Economica, 1991.

SOLEIL, Sylvain. Pourquoi comparait-on les droits au XIX ${ }^{\mathrm{e}}$ siècle. Revue Clio@Themis, [s. l.], n. 13, p. 01-21, 2017.

TALON, D. Histoire du droit et droit comparé: une alliance féconde. In: BONTEMS, Claude. Nonagesimo Anno: mélanges en hommage à Jean Gaudemet. Paris: Presses Universitaires de France, 1999. p. 629 et seq.

ZIMMERMANN, Reinhard. L'héritage de Savigny. Histoire du droit, droit comparé, et émergence d'une science juridique européenne. Revue internationale de droit économique, [s. l.], t. XXVII, n. 1-2, p. 95-127, 2013.

ZLATESCU, Victor Dan. Quelques aspects méthodologiques de la comparaison des droits. Revue internationale de droit comparé, [s. l.], v. 35, n. 3, p. 559-566, 1983.

\section{DADOS DA PUBLICAÇÃO}

Categoria: artigo de autor convidado.

Recebido em: 03/12/2021.

Aceito em: 13/12/2021. 